\title{
伴有边界摄动的非线性 Robin 边值问题的奇摄动
}

\author{
林宗池 \\ (福越师范大学数学系, 福州) \\ 林 苏 標 \\ (福建电大数学科,檑州)
}

本文研究非线性 Robin 边值问题.

$$
\begin{aligned}
& \varepsilon y^{\prime \prime}=f\left(x, y, y^{\prime}, \varepsilon, \mu\right), \mu<x<1-\mu, \\
& \left.a_{1}(\varepsilon, \mu) y(x, \varepsilon, \mu)\right|_{x=\mu}-\left.b_{1}(\varepsilon, \mu) y^{\prime}(x, \varepsilon, \mu)\right|_{x=\mu}=\varphi_{1}(\varepsilon, \mu), \\
& \left.a_{\delta}(\varepsilon, \mu) y(x, \varepsilon, \mu)\right|_{x=1-\mu}+\left.b_{2}(\varepsilon, \mu) y^{\prime}(x, \varepsilon, \mu)\right|_{x=1-\mu}-\varphi_{2}(\varepsilon, \mu)
\end{aligned}
$$

的奇摄动. 其中 $\varepsilon 、 \mu$ 是两个正的小参数， $a_{i} 、 b_{i} 、 \varphi_{i}(i=1,2)$ 为 $\varepsilon, \mu$ 的充分光滑的函数, 且 $a_{i}^{2}+b_{i}^{2} \neq 0$. 为方便起见, 设 $a_{i}>0, b_{1}>0, b_{2}(0,0)=0$. 当 $a_{i}=1, b_{i}=0(i=$ $1,2)$ 时, 章国华和林宗池 ${ }^{[1]}$ 曾研究了由外解展开到任意项和内解 (即边界层校正函数)只展开 到一项而构成的解的存在并作出余项的估计. 本文在文献 [1] 的基础上, 研究在更一般的边 界条件下, 由外解和内解同时展开到任意项而构成的解存在并对余项作出估计,改进和拓广了 文献 [1] 的工作.

首先, 我们构造问题 (1)-(3) 的外解. 鉴于问题 (1)-(3) 包含两个形式参数,因此, 假 定解也具有两个参数的如下的形式的渐近展开式:

$$
Y(x, \varepsilon, \mu) \sim y_{0,0}(x)+\sum_{i=1}^{\infty} \sum_{i=0}^{i} y_{i-i, j}(x) \varepsilon^{i-i} \mu^{i},
$$

其中 $y_{i-i, j}(x),(i=0,1, \cdots, i ; i=1,2, \cdots)$ 是待定的函数. 把 (4) 式代人 (1) 式, 我们 有

$$
\varepsilon \frac{d^{2} Y}{d x^{2}}=f\left(x, \mathrm{Y}, \mathrm{Y}^{\prime} \varepsilon, \mu\right)
$$

设 $f_{Y^{\prime}}, \leqslant-k<0$ ( $k$ 为某一正常数)、当 $\varepsilon=\mu=0$ 时, 摄动问题退化为非摄动问题:

$$
\begin{aligned}
& f\left(x, Y, Y_{0}^{\prime} 0,0\right)=0,0<x<1, \\
& a_{2}(0,0) Y(1,0,0)=\varphi_{2}(0,0),
\end{aligned}
$$

它的解用 $y_{0,0}(x)$ 表示.

又假设 $f\left(x, Y, Y^{\prime} \varepsilon, \mu\right), a_{i}, b_{i}, \varphi_{i}(i=1,2)$ 当两参数 $\varepsilon, \mu$ 都趋于零时, 均有关于 $8, \mu$ 的渐近的二重级数展开式(参看文献 [2-4]):

$$
\begin{aligned}
& a_{i}(\varepsilon, \mu) \sim \sum_{i=0}^{\infty} \sum_{i=0}^{i} a_{i}^{(i-i, i)} \varepsilon^{i-i} \mu^{i},(i=1,2), \\
& b_{i}(\varepsilon, \mu) \sim \sum_{i=0}^{\infty} \sum_{i=0}^{i} b_{i}^{(i-i, i)} \varepsilon^{i-i} \mu^{i},\left(i=1,2 ; b_{2}(0,0)-0\right),
\end{aligned}
$$

本文 1986 年 7 月 23 日收到. 


$$
\begin{aligned}
& \varphi_{i}(\varepsilon, \mu) \sim \sum_{i=0}^{\infty} \sum_{j=0}^{i} \varphi_{i}^{(i-i, j)} \varepsilon^{i-i} \mu^{i},(i=1,2), \\
& f\left(x, Y, Y_{,}^{\prime}, \varepsilon, \mu\right) \equiv F(\varepsilon, \mu) \sim F(0,0)+\sum_{i=1}^{\infty} \sum_{i=0}^{i} F_{i-i, j}(0,0) \varepsilon^{i-i} \mu^{\prime},
\end{aligned}
$$

其中 $\quad F(0,0)-f\left(x, y_{0,0}, y_{0,0}^{\prime}, 0,0\right), F_{i-i, j}(0,0)=f_{Y}\left(x, y_{0,0}, y_{0,0}^{\prime}, 0,0\right) y_{i-i, j}$

$$
+f_{y^{\prime}}\left(x, y_{0,0}, y_{0,0}^{\prime}, 0,0\right) y_{i-j, j}^{\prime}+G_{i-j, j}(j-0,1, \ldots, i ; i=1,2, \cdots) \text {. }
$$

$G_{l, k}$ 由 $x, y_{l, k}, y_{l, k}^{\prime}(0 \leqslant l \leqslant i-j-1 ; 0 \leqslant k \leqslant j-1)$ 确定.

上述记号适用于其他的展式.

考虑到 (4)、(6) 和 (11) 式,由 (5) 式得

$$
f_{y} y_{i-i, j}^{\prime}+f_{y} y_{i-i, j}=y_{i-i-1}^{\prime \prime}-G_{i-j, j}(j-0,1, \cdots, i ; i=1,2, \cdots) .
$$

为了求得 $y_{i-j, j}(x)$, 我们需要适当的边界条件 $y_{i-j, j}(1)$, 它们将在下面给出. 把求得 的 $y_{i-j, j}(x)$ 代人 (4) 式,我们就得到问题 (1)一-(3) 外解的形式展开式.一般地,它不满足边 界条件 (2). 因此, 我们将在 $x=\mu$ 点构造边界层函数. 令

$$
\bar{Y}=Y(x, \varepsilon, \mu)+W(t, \varepsilon, \mu),
$$

其中 $t=\frac{x-\mu}{8}$ 为伸长变量. 我们假设 $W(t, 8, \mu)$ 有如下的形式展开式:

$$
W(t, 8, \mu) \sim 8 \sum_{i=0}^{\infty} \sum_{i=0}^{i} w_{i-i, j}(t) 8^{i-i} \mu^{i},
$$

把 (13) 式代人 (1) 式,我们有

$$
8 \frac{d^{2} \bar{Y}}{d x^{2}}=f\left(x, \bar{Y}, \bar{Y}^{\prime}, 8, \mu\right) .
$$

从 (4)、(5) 和 (13) 式, 我们有

$$
\begin{aligned}
8^{-1} \frac{d^{2} W}{d t^{2}} & =f\left(\mu+\varepsilon t, Y+W, Y_{x}^{\prime}+8^{-1} W_{t}^{\prime}, \varepsilon, \mu\right)-f\left(\mu+\varepsilon t, Y, Y_{x}^{\prime}, 8, \mu\right) \\
& \equiv \tilde{F}(\varepsilon, \mu)-\tilde{F}(0,0)+\sum_{i=1}^{\infty} \sum_{j=0}^{j} \tilde{F}_{i-i, j}(0,0) \varepsilon^{i-i} \mu^{i},
\end{aligned}
$$

其中

$$
\begin{aligned}
F(0,0) & =f\left(0, y_{0,0}(0), y_{0,0}^{\prime}(0)+\frac{d w_{0,0}(t)}{d t}, 0,0\right)-f\left(0, y_{0,0}(0), y_{0,0}^{\prime}(0), 0,0\right) \\
& =f_{Y \prime}\left(0, y_{0,0}(0), y_{0,0}^{\prime}(0)+\theta_{1} \frac{d w_{0,0}(t)}{d t}, 0,0\right) \frac{d w_{0,0}(t)}{d t}, 0<\theta_{1}<1 ; \\
\tilde{F}_{i-j, j}(0,0) & -\frac{1}{(i-j) ! j !} \frac{\partial^{i} \tilde{F}(\varepsilon, \mu)}{\left.\partial \varepsilon^{i-i \partial \mu^{i}}\right|_{s=\mu-0}} \\
& -f_{Y \prime}\left(0, y_{0,0}(0), y_{0,0}^{\prime}(0)+\frac{d w_{0,0}(t)}{d t}, 0,0\right) \frac{d w_{i-j, j}(t)}{d t}+C_{i-j, j}(t),
\end{aligned}
$$

这里的 $C_{i-j, j}(t)$ 可以由 $w_{r, p}(t)$ 和 $w_{r, s}^{\prime}(t),(0 \leqslant r \leqslant i-i ; 0 \leqslant s \leqslant j-1)$ 逐步确定.

把 (14) 式代人 (16) 式的左端, 比较同 $\varepsilon, \mu$ 次常的系数, 得

$$
\frac{d^{2} w_{0,0}}{d t^{2}}-f_{y^{\prime}}\left(0, y_{0,0}(0), y_{0,0}^{\prime}(0)+\theta_{1} \frac{d w_{0,0}(t)}{d t}, 0,0\right) \frac{d w_{0,0}}{d t},
$$




$$
\begin{gathered}
\frac{d^{2} w_{i-j, j}}{d t^{2}}=f_{y}\left(0, y_{0,0}(0), y_{0,0}^{\prime}(0)+\frac{d w_{0,0}(t)}{d t}, 0,0\right) \frac{d w_{i-i, j}}{d t}+C_{i-j, j}(t) \\
(j=0,1, \cdots, i ; i-1,2, \cdots),
\end{gathered}
$$

其中负下标的量取作零.

为了从 (17)、(18) 式解得 $W_{i-i, j}(t),(j=0,1, \cdots, i ; i=0,1, \cdots)$, 我们也要在下面 给出关于 $w_{i-j,-j}$ 的初始条件.

现在我们来确定 $y_{i-j, i}$ 与 $w_{i-i, i},(j=0,1, \ldots, i ; i=0,1, \cdots)$ 的边界条件和初始条 件. 把 (4)、(8)-(10)、(13)、(14) 式代人 (2) 和 (3) 式, 并把 $y_{i-i, j}(\boldsymbol{\mu}), \boldsymbol{y}_{i-j, j}(1-\boldsymbol{\mu})$ 在 $\boldsymbol{\mu}=0$ 点按 $T$ aylor 公式展开,比较同 $\varepsilon, \mu$ 次幕的系数,得

$$
\begin{aligned}
& a_{2}^{(0,0)} y_{i-i, j}(1)=\varphi_{2}^{(i-i, i)}-a_{2}^{(0,0)} \sum_{s=1}^{1} \frac{(-1)^{s}}{s !} y_{i-i, j-s}^{(s)}(1) \\
& -a_{2}^{(1,0)} \sum_{s=0}^{j} \frac{(-1)^{s}}{s !} y_{i-i-1, i-s}^{(s)}(1)-a_{i}^{(0,1)} \sum_{s=0}^{j-1} \frac{(-1)^{s}}{s !} y_{i-i, j-1-s}^{(s)}(1)+\cdots \\
& +a_{2}^{(1-i, i)} y_{0,0}(1)-b_{2}^{(1,0)} \sum_{s=0}^{j} \frac{(-1)^{s}}{s !} y_{i-i-1, j-s}^{(s)} \\
& -b_{2}^{(0,1)} \sum_{s=0}^{j-1} \frac{(-1)^{s}}{s !} y_{i-i, j-1-s}^{(s)}(1)+\cdots+b_{2}^{(i-i, j)} y_{0,0}(1) \\
& -\sum_{s=0}^{i-j-1} \sum_{k=0}^{j} a_{2}^{(s, k)} W_{i-j-1-s, j-k}(0) \\
& -\sum_{s=1}^{i-j} \sum_{k=0}^{i} b_{2}^{(s, k)} w_{i-i-s, i-k}^{\prime}(0)-b_{2}^{(0,1)} w_{i-i, i-1}^{\prime}(0) \\
& (j=0,1, \ldots, i ; i=1,2, \cdots) \text {, } \\
& b_{1}^{(0,0)} w_{i-i, j}^{\prime}(0)=-\varphi_{1}^{(i-i, j)}+\sum_{s=1}^{i-j} \sum_{k=0}^{j} b_{1}^{(s, k)} w_{i-j-s, j-k}^{\prime}(0) \\
& +\int a_{1}^{(0,0)} \sum_{s=0}^{j} \frac{1}{s !} y_{i-i, j-s}^{(s)}(0)+a_{1}^{(1,0)} \sum_{s=0}^{j} \frac{1}{s !} y_{i-i-1, j-s}^{(s)}(0) \\
& \left.+\cdots+a_{1}^{(i-i, j)} y_{0,0}(0)\right]+\sum_{r=0}^{i-j-s} \sum_{s=0}^{j} a_{1}^{(r, s)} w_{i-j-1-r, j-s}(0) \\
& -\left[b_{1}^{(0,0)} \sum_{s=0}^{j} \frac{1}{s !} y_{i-i, i-s}^{(s+1)}(0)+b_{1}^{(1,0)} \sum_{s=0}^{j} y_{i-j-1, j-s}^{(s+1)}(0)\right. \\
& \left.+\cdots+b_{1}^{(i-i, i)} w_{0,0}^{\prime}(0)\right] \text {. }
\end{aligned}
$$

从 (6)、(7)、(12)、(17)-(20) 式我们能够依次求得 $y_{0,0}(x), w_{0,0}(t), y_{i-j, j}(x), w_{i-j, i}(t)$ $(j=0,1, \cdots, i ; i=1,2, \cdots)$ 类似于文献 [5], 我们求得 $w_{i-j, j}(t)$ 有如下的估计式:

$$
\begin{gathered}
w_{0,0}(t)-O\left(e^{-k t}\right), \\
w_{i-i, j}(t)=O\left(e^{-k\left(1-\delta_{i-i \cdot j}\right) t}\right),(t \gg 1, i=0,1, \cdots, i ; i=1,2, \cdots)
\end{gathered}
$$

这里的 $\delta_{i-j, j}$ 是小正数.

于是我们有如下的定理.

定理 1 假定下面条件成立: 
a) 退化问题 (6)、(7) 有一个解 $y_{0,0}(x) \in c^{(2)}([0,1])$;

b) 函数 $f\left(x, y, y^{\prime}, \varepsilon, \mu\right) \in c^{(m+1)}(D)$, 其中 $D-\left\{\mu \leqslant x \leqslant 1-\mu,\left|y-y_{0,0}\right| \leqslant d_{1}\right.$, $\left.\left|y^{\prime}\right|<\infty, 0 \leqslant \varepsilon \leqslant \varepsilon_{1}, 0 \leqslant \mu \leqslant \mu_{1}\right\},\left(\varepsilon_{1}, \mu_{1}, d_{1}\right.$ 为三个小正数);

c) 存在两个正常数 $l, k$, 使得在 $D$ 上 $f_{Y} \geqslant l, f_{Y^{\prime}} \leqslant-k<0$;

d) 函数 $t$ 在 $D$ 上满足 Nagumo 条件, 即存在一个定义在 $[0,+\infty)$ 上正的连续函数 $\varphi$, 使当 $s \rightarrow \infty$ 时, $s^{2} / \varphi(s) \rightarrow \infty$ 和 $\left|f\left(x, y, y^{\prime}, \varepsilon, \mu\right)\right| \leqslant \varphi\left(\left|y^{\prime}\right|\right)$;

e) $a_{i}(\varepsilon, \mu), b_{i}(\varepsilon, \mu), \varphi_{i}(\varepsilon, \mu) \in C^{(m+1)}\left(0 \leqslant \varepsilon \leqslant \varepsilon_{1} ; 0 \leqslant \mu \leqslant \mu_{1}\right)$ 且 $a_{i}>0, b_{1}>0$, $b_{2}(0,0)=0$, 则 Robin 边值问题 (1)-(3) 存在一个解 $y(x, \varepsilon, \mu)$ 和下列估计式成立:

$$
y(x, \varepsilon, \mu)-Z_{m}=O\left(\sum_{i=0}^{m+1} \varepsilon^{m+1-i} \mu^{i}\right),
$$

其中 $Z_{m}-Y_{m}(x, \varepsilon, \mu)+W_{m}(t, \varepsilon, \mu)-\sum_{i=0}^{m} \sum_{i=0}^{i}\left(y_{i-i, i}(x)+\varepsilon w_{i-i, i}(t) \varepsilon^{i-i} \mu^{\prime}\right.$.

证 对于 $[\mu, 1-\mu]$ 中的 $X$ 和充分小的 $\varepsilon>0, \mu>0$, 我们构造下列两个函数:

$$
\alpha(x, \varepsilon, \mu)=Z_{m}-\sum_{i=0}^{m+1} \varepsilon^{m+1-i} \mu^{i} r l^{-1}, \beta(x, \varepsilon, \mu)=Z_{m}+\sum_{i=0}^{m+1} \varepsilon^{m+1-i} \mu^{i} r l^{-1},
$$

其中 $Z_{m}$ 如上所求, $r$ 是待定的正常数.

显然,

$$
\begin{aligned}
& \alpha(x, \varepsilon, \mu) \in c^{(2)}([\mu, 1-\mu]) \subset c^{(2)}([0,1]), \\
& \beta(x, \varepsilon, \mu) \in c^{(2)}([\mu, 1-\mu]) \subset c^{(2)}([0,1])
\end{aligned}
$$

和

$$
\begin{aligned}
& a_{1}(\varepsilon, \mu) \alpha(\mu, \varepsilon, \mu)-b_{1}(\varepsilon, \mu) \alpha^{\prime}(\mu, \varepsilon, \mu) \leqslant \varphi_{1}(\varepsilon, \mu) \\
& \quad \leqslant a_{1}(\varepsilon, \mu) \beta(\mu, \varepsilon, \mu)-b_{1}(\varepsilon, \mu) \beta^{\prime}(\mu, \varepsilon, \mu), \\
& a_{2}(\varepsilon, \mu) \alpha(1-\mu, \varepsilon, \mu)+b_{2}(\varepsilon, \mu) \alpha^{\prime}(1-\mu, \varepsilon, \mu) \leqslant \varphi_{2}(\varepsilon, \mu) \\
& \quad \leqslant a_{2}(\varepsilon, \mu) \beta(1-\mu, \varepsilon, \mu)+b_{2}(\varepsilon, \mu) \beta^{\prime}(1-\mu, \varepsilon, \mu) .
\end{aligned}
$$

利用中值定理, 不难验证, 当 $r=\max \left\{\delta, r_{0}\right\}$ 时, $\left(\delta, r_{0}\right.$ 为两个足够大的正数), 佰有

$$
\varepsilon \alpha^{\prime \prime} \geqslant f\left(x, \alpha, \alpha^{\prime}, \varepsilon, \mu\right), \varepsilon \beta^{\prime \prime} \leqslant f\left(x, \beta, \beta^{\prime}, \varepsilon, \mu\right), \mu<x<1-\mu,
$$

因此,利用 Nagumo 定理 ${ }^{[6,7]}$, 问题 (1)一(3) 存在一个解 $y(x, \mu, \varepsilon) \in C^{(2)}(D)$, 满足

$$
\alpha(x, \varepsilon, \mu) \leqslant y(x, \varepsilon, \mu) \leqslant \beta(x, \varepsilon, \mu) .
$$

定理 1 证毕.

注. 当 $f_{y^{\prime}} \geqslant k>0$ 时, 在相应的假设下, 可得到类似的结果,但这时的边界层校正项 有如下的估计式: $V_{i-j, j}(\tau)=O\left(e^{-k\left(1-s_{i-i, j}\right) \tau}\right), \quad(i=0,1, \cdots, i ; i=0,1, \cdots, m)$, 而伸 长变星

$$
\tau=\frac{1-\mu-x}{\varepsilon}
$$

\section{考文姑}

[1] 章国华、林宗池,应用数学和力学, 5(1984), 5: 603-612.

[2] O'Malley, R. E., Arch. Rational. Mech. Anal. 40 (1971), $209-222$.

[3] 林宗池, 应用数学和力学, 3(1982)，1: 33-48; 5: 641-652; 5(1984), 3: 363-375.

[4] 林宗他,应用数学学报, 7(1984), 1: 124-128.

[5] O'Malley, R. F., Introduction to Singular Perturbations, Academic Prex, New York, 1974.

[6] Nagumo, M., Proc. Phys. Math. Soc. Japan, 19 (1937), 861-866.

[7] Heidel, J. M., J. of Math Anal. and Appl., 48(1974), 493-503. 